\title{
LAMB'S PLANE PROBLEM IN A THERMO-ELASTIC MICROPOLAR MEDIUM WITH STRETCH
}

\section{T.K. CHADHA and RAJNEESH KUMAR}

\author{
Department of Mathematics \\ Guru Nanak Dev University \\ Amritsar, India \\ and
}

\section{LOKENATH DEBNATH}

Department of Mathematics University of Central Florida Orlando, Florida 32816, USA

(Received May 23, 1984 and in revised form January 8, 1985)

ABSTRACT. A study is made of the Lamb plane problem in a thermo-elastic micropolar medium with the effect of stretch. The problem is solved for an arbitrary, normal load distribution by using the double Fourier transform. The displacement components, force stress, couple stress, vector first moment and the temperature field are determined for a half space subjected to an arbitrary normal load. Two special cases of a horizontal force and a torque which are oscillating with a frequency $\omega$ have been investigated. It is shown that results of this analysis reduce to those without stretch.

KEY WORDS AND PHRASES. Lamb's problem, Fourier transform, and micropolar medium.

1980 MATHEMATICS SUBJECT CLASSIFICATION CODE. $\quad 73 D 30$

\section{INTRODUCTION.}

Eringen and Suhubi [1] have developed a general theory of linear and nonlinear micro-elastic continua. This theory contains the cosserat continuum and the intermediate couple stress theories as special cases. In a subsequent paper [2], Eringen recapitulated his work and renamed his theory as micropolar elasticity. The micropolar theory essentially deals with such materials whose constituents are dumbel1 type molecules and are allowed to rotate independently without stretch. Later on, Eringen [3-4] extended his work to include the effect of axial stretch during the rotation of molecules and developed theories for both micropolar elastic solids with stretch and micropolar fluids with stretch. The mechanical model underlying the theory of micropolar elastic solids with stretch can be envisioned as an elastic medium composed of a large number of short springs. These springs possess average inertia and can deform in axial directions. 
Lamb's problem [5] has been investigated extensively by several researchers in different elastic media with various kinds of loading. In particular, Nowacki and Nowacki [6] have studied the Lamb problem in micropolar elastic media. Recently, Chadha [7] has investigated the same problem in micropolar elastic media, and discussed wave propagation in a semi-infinite micropolar elastic solid due to loading at the plane boundary of semi-half space. Acharya and Sengupta [8] have recently studied Lamb's problen in a thermo-elastic medium under the influence of temperature. They have examined the longitudinal and transverse thermo-elastic wave propagation in a micropolar semi-infinite space bounded by a plane in which a normal loading is applied.

In spite of these studies, no attention is given to Lamb's problem in thermo-micropolar elastic half-space with stretch. The main purpose of this paper is to investigate the problem with the assumption that the heat is radiated from the free plane boundary surface of the semi-infinite space and the maximum temperature difference across the surface is always small. The displacement components, force stress, couple stress, vector first moment and the temperature field are determined for the half-space subjected to an arbitrary normal load. Two special cases of a horizontal force and a torque which are harmonic in time have been discussed. The problem is solved by the double Fourier transform method.

2. THE FORMULATION OF THE PROBLEM AND THE BOUNDARY CONDITIONS

We consider a homogeneous micropolar elastic semi-infinite space with stretch under the influence of temperature. We assume that there is a uniform stretch in the $x$-direction only and a loading $g(x, t)$ normal to the free boundary surface $z=0$. Further, we assume that the micropolar semi-space is free to exchange heat within the region $z>0$; and prior to the appearance of any disturbance, both media are everywhere at the constant absolute temperature $T_{0}$.

We consider the two-dimensional problem so that the displacement and rotation are independent of the $y$ coordinate. Thus we may write $\underline{u}=\left(u_{1}, 0, u_{3}\right)$ and $\underline{\omega}=\left(0, \omega_{2}, 0\right)$. The displacements are related to the displacement potentials $\phi(x, z, t)$ and $\psi(x, z, t)$ as follows:

$$
u_{1}=\frac{\partial \phi}{\partial x}+\frac{\partial \psi}{\partial z}, \quad u_{3}=\frac{\partial \phi}{\partial z}-\frac{\partial \psi}{\partial x}
$$

so that

$$
e=\nabla^{2} \phi, \quad \nabla^{2} \psi=\frac{\partial u_{1}}{\partial z}-\frac{\partial u_{3}}{\partial x}
$$

where

$$
\nabla^{2} \equiv \frac{\partial^{2}}{\partial x^{2}}+\frac{\partial^{2}}{\partial z^{2}} \quad \text { and } \quad e=\frac{\partial u_{1}}{\partial x}+\frac{\partial u_{3}}{\partial z} \text {. }
$$

We follow Eringen [4] and Nowacki [9] to write down the basic field equations in a thermo-micropolar elastic solid medium with stretch and without body forces and body moments. These field equations are

$$
\begin{aligned}
& (\mu+\alpha) \nabla^{2} \underline{u}+(\lambda+\mu-\alpha) \operatorname{grad} \operatorname{div} \underline{u}+2 \alpha \operatorname{rot} \underline{\omega}-v \operatorname{grad} \theta=\rho \frac{\partial^{2} \underline{u}}{\partial t^{2}}, \\
& (\gamma+\varepsilon) \nabla^{2} \underline{\omega}+(\gamma+\beta-\varepsilon) \text { grad div } \underline{\omega}-4 \alpha \underline{\omega}+2 \alpha \operatorname{rot} \underline{u}=J \frac{\partial^{2} \underline{\omega}}{\partial t^{2}},
\end{aligned}
$$




$$
\alpha_{0} \nabla^{2} \Phi-\eta_{0} \Phi=\frac{J}{2} \frac{\partial^{2} \Phi}{\partial t^{2}},
$$

where $\lambda, \mu, \alpha, \beta, \gamma, \varepsilon, \alpha_{0}, \eta_{0}$ are material constants, $\rho$ is the density of the material, $J$ is the rotational inertia, $\nu=(3 \lambda+2 \mu) \alpha_{t}, \alpha_{t}$ is the coefficient of linear expansion of the solid, $\theta=T-T_{0}=$ absolute temperature minus the initial absolute temperature $T_{0}$.

Using the values of $\underline{u}$ and $\underline{\omega}$ in equations (2.4)-(2.6) we get

$$
\begin{gathered}
(\mu+\alpha) \nabla^{2} u_{1}+(\lambda+\mu-\alpha) \frac{\partial e}{\partial x}-2 \alpha \frac{\partial \omega_{2}}{\partial z}-v \frac{\partial \theta}{\partial x}=\rho \frac{\partial^{2} u_{1}}{\partial t^{2}}, \\
(\mu+\alpha) \nabla^{2} u_{3}+(\lambda+\mu-\alpha) \frac{\partial e}{\partial z}+2 \alpha \frac{\partial \omega_{2}}{\partial z}-v \frac{\partial \theta}{\partial z}=\rho \frac{\partial^{2} u_{3}}{\partial t^{2}}, \\
(\gamma+\varepsilon) \nabla^{2} \omega_{2}-4 \alpha \omega_{2}+2 \alpha\left(\frac{\partial u_{1}}{\partial z}-\frac{\partial u_{3}}{\partial x}\right)=J \frac{\partial^{2} \omega_{2}}{\partial t^{2}}, \\
\alpha_{0} \nabla^{2}-n_{0} \Phi=\frac{J}{2} \frac{\partial^{2} \Phi}{\partial t^{2}} .
\end{gathered}
$$

The temperature field $\theta(x, z, t)$ satisfies Fourier's Law of heat conduction, which in the present case can be written as

$$
\kappa \nabla^{2} \theta=\rho C \frac{\partial^{2} \theta}{\partial t^{2}}+T_{0} \nu \frac{\partial}{\partial t}\left(\nabla^{2} \phi\right)
$$

where $k$ is thermal conductivity and $C$ is the specific heat at constant strain. Using (2.1ab)-(2.3ab) and (2.11) in equations (2.7)-(2.10), we obtain

$$
\begin{aligned}
& \left(\nabla^{2}-\frac{1}{c_{1}^{2}} \frac{\partial^{2}}{\partial t^{2}}\right) \phi-q \theta=0, \\
& \left(\nabla^{2}-\frac{1}{c_{3}^{2}} \frac{\partial}{\partial t}\right) \theta-r \frac{\partial}{\partial t}\left(\nabla^{2} \phi\right)=0, \\
& \left(\nabla^{2}-\frac{1}{c_{2}^{2}} \frac{\partial^{2}}{\partial t^{2}}\right) \psi-p \omega_{2}=0, \\
& \left(\nabla^{2}-\gamma_{1}^{2}-\frac{1}{c_{4}^{2}} \frac{\partial^{2}}{\partial t^{2}}\right) \omega_{2}+s \nabla^{2} \psi=0, \\
& \left(\nabla^{2}-r_{2}^{2}-\frac{1}{c_{5}^{2}} \frac{\partial^{2}}{\partial t^{2}}\right) \Phi=0,
\end{aligned}
$$

where

$$
c_{1}^{2}=\frac{\lambda+2 \mu}{\rho}, c_{2}^{2}=\frac{\mu+\alpha}{\rho}, c_{3}^{2}=\frac{K}{\rho C}, c_{4}^{2}=\frac{\gamma+\varepsilon}{J}, c_{5}^{2}=\frac{2 \alpha_{0}}{J}
$$




$$
\begin{gathered}
\gamma_{1}^{2}=\frac{4 \alpha}{\gamma+\varepsilon}, \gamma_{2}^{2}=\frac{n_{0}}{\alpha_{0}}, p=\frac{2 \alpha}{\mu+\alpha}, q=\frac{\nu}{\lambda+2 \mu}, \\
r=\frac{T_{0} \nu}{\kappa}, s=\frac{2 \alpha}{\gamma+\varepsilon} .
\end{gathered}
$$

We next eliminate $\phi$ or $\theta$ from equations (2.12)-(2.13), and $\psi$ or $\omega_{2}$ from (2.14)-(2.15) to obtain the following partial differential equations:

$$
\begin{gathered}
{\left[\left(\nabla^{2}-\frac{1}{c} \frac{\partial^{2}}{\partial t^{2}}\right)\left(\nabla^{2}-\frac{1}{c_{3}^{2}} \frac{\partial}{\partial t}\right)-\tau^{2} \frac{\partial}{\partial t} \nabla^{2}\right](\phi, \theta)=0,} \\
{\left[\left(\nabla^{2}-\frac{1}{2} \frac{\partial^{2}}{\partial t^{2}}\right)\left(\nabla^{2}-r_{1}^{2}-\frac{1}{c_{4}^{2}} \frac{\partial^{2}}{\partial t^{2}}\right)+\zeta^{2} \nabla^{2}\right]\left(\psi, \omega_{2}\right)=0,}
\end{gathered}
$$

where $\tau^{2}=q r, \zeta^{2}=p s$.

Following Eringen [4] and Nowacki [9] the stress tensor $\sigma_{j i}$ and the couple stress tensor $\mu_{j i}$ are given by

$$
\begin{aligned}
& \sigma_{j i}=\left(\lambda u_{k, k}-v \theta\right) \delta_{i j}+(\mu-\alpha)\left(u_{i, j}+u_{j, i}\right)+2 \alpha\left(u_{i, j}-\varepsilon_{k j i} \omega_{k}\right), \\
& \mu_{j i}=\beta_{0} \varepsilon_{k j i} \Phi, k+\beta \omega_{k, k} \delta_{i j}+(\gamma-\varepsilon) \omega_{j, i}+(\gamma+\varepsilon) \omega_{i, j}, \\
& \beta_{j}=\alpha_{0} \Phi, j+\frac{1}{3} \beta_{0} \varepsilon_{k j i} \omega_{k, i},
\end{aligned}
$$

where $\varepsilon_{j k i}$ is unit antisymmetric tensor, $\beta_{j}$ is the vector first moment and $i, j, k=1,2,3$. These expressions in the present case reduce to the form

$$
\begin{aligned}
& \sigma_{33}=2 \mu\left(\frac{\partial^{2} \phi}{\partial z^{2}}-\frac{\partial^{2} \psi}{\partial x \partial z}\right)+\lambda \nabla^{2} \phi-\nu \theta, \\
& \sigma_{31}=\mu\left[2 \frac{\partial^{2} \phi}{\partial x \partial z}+\frac{\partial^{2} \psi}{\partial z^{2}}-\frac{\partial^{2} \psi}{\partial x^{2}}\right]+\alpha\left(\nabla^{2} \psi-2 \omega_{2}\right), \\
& \mu_{32}=(\gamma+\varepsilon) \frac{\partial \omega_{2}}{\partial z}-\beta_{0} \frac{\partial \Phi}{\partial x}, \\
& \beta_{3}=\alpha_{0} \frac{\partial \Phi}{\partial z}+\frac{1}{3} \beta_{0} \frac{\partial \omega_{2}}{\partial x} .
\end{aligned}
$$

3. BOUNDARY CONDITIONS.

In view of the normal loading of magnitude $g(x, t)$ applied on $z=0$, the boundary conditions are given by

$$
\sigma_{33}=-g(x, t), \sigma_{31}=0, \mu_{32}=0, \beta_{3}=0 \text {, at } z=0
$$

In view of the assumption that the temperature difference across the free surface is always small, the linearized form of the radiation condition is valid on the boundary $z=0$ so that

$$
\frac{\partial \theta}{\partial z}+h \theta=0 \quad \text { on } z=0
$$

Further, if we assume that the loading function $g(x, t)$ is bounded and finite on $z=0$, then $\phi, \psi, \theta, \omega_{2}$ and $\Phi$ vanish at infinity. 


\section{SOLUTION OF THE PROBLEM.}

We solve the above equations (2.16), (2.20) and (2.21) by using the double Fourier transform defined as follows:

$$
\bar{f}(k, z, n)=\frac{1}{2 \pi}-_{-\infty}^{\infty} \int_{-\infty}^{\infty} f(x, z, t) e^{i(k x+n t)} d x d t,
$$

where the inverse transform is given by

$$
f(x, z, t)=\frac{1}{2 \pi}-\infty \int_{-\infty}^{\infty} \int^{\infty} \bar{f}(k, z, n) e^{-i(k x+n t)} d k d n .
$$

Thus the equations reduce to the form

$$
\begin{aligned}
\left(\frac{d^{2}}{d z^{2}}-\lambda_{1}^{2}\right)\left(\frac{d^{2}}{d z^{2}}-\lambda_{2}^{2}\right)(\bar{\phi}, \bar{\theta}) & =0, \\
\left(\frac{d^{2}}{d z^{2}}-\lambda_{3}^{2}\right)\left(\frac{d^{2}}{d z^{2}}-\lambda_{4}^{2}\right)\left(\bar{\psi}, \bar{\omega}_{2}\right) & =0, \\
\left(\frac{d^{2}}{d z^{2}}-\lambda_{5}^{2}\right) \Phi & =0,
\end{aligned}
$$

where

$$
\begin{aligned}
\lambda_{1}^{2}+\lambda_{2}^{2} & =\left(2 k^{2}-\frac{n^{2}}{c_{1}^{2}}-\frac{i n}{c_{3}^{2}}-i n \tau^{2}\right), \\
\lambda_{1}^{2} \lambda_{2}^{2} & \left.=\left(\frac{n^{2}}{c_{1}^{2}}-k^{2}\right)\left(\frac{i n}{c_{3}^{2}}-k^{2}\right)-i n \tau^{2} k^{2}\right), \\
\lambda_{3}^{2}+\lambda_{4}^{2} & =\left(2 k^{2}-\frac{n^{2}}{c_{2}^{2}}-\frac{n^{2}}{c_{4}^{2}}+r_{1}^{2}-k^{2}\right), \\
\lambda_{3}^{2} \lambda_{4}^{2} & =\left(\frac{n^{2}}{c_{2}^{2}}-k^{2}\right)\left(\frac{n^{2}}{c_{4}^{2}}-k^{2}-r_{1}^{2}\right)-k^{2} \zeta^{2}, \\
\lambda_{5}^{2} & =k^{2}+r_{2}^{2}-\frac{n^{2}}{c_{5}^{2}} .
\end{aligned}
$$

In view of the boundary conditions at infinity, the bounded solutions of (4.3)(4.5) assume the form

$$
\begin{aligned}
& \bar{\phi}=A e^{-\lambda 1^{z}}+B e^{-\lambda 2^{z},} \\
& \bar{\theta}=A_{1} e^{-\lambda 1} 1^{2}+B_{1} e^{-\lambda 2^{z}}, \\
& \bar{\psi}=C e^{-\lambda 3^{z}}+D e^{-\lambda} 4^{z}, \\
& \bar{\omega}_{2}=C_{1} e^{-\lambda 3^{z}}+D_{1} e^{-\lambda} 4^{z}, \\
& \Phi=E e^{-\lambda 5^{z}},
\end{aligned}
$$

where

$$
A_{1}=\alpha_{1} A, B=\alpha_{2} B, C=\alpha_{3} C, D_{1}=\alpha_{4} D,
$$


and

$$
\alpha_{j}=\left[\begin{array}{ll}
\frac{1}{q}\left(\lambda_{j}^{2}+\frac{\eta^{2}}{c_{1}^{2}}-k^{2}\right), & \text { for } j=1,2 . \\
\frac{1}{p}\left(\lambda_{j}^{2}+\frac{n^{2}}{c_{2}^{2}}-k^{2}\right), & \text { for } j=3,4 .
\end{array}\right]
$$

It is assumed that $\operatorname{Re}\left(\lambda_{j}\right) \geq 0, j=1,2,3,4,5$.

Applying the Fourier transform (4.1) to (3.1abcd)-(3.2) and using (2.25)-(2.28), it turns out that

$$
\begin{gathered}
2 \mu\left\{\frac{d^{2} \bar{\phi}}{d z^{2}}+i k \frac{d \bar{\psi}}{d z}\right\}+\lambda\left(\frac{d^{2}}{d z^{2}}-k^{2}\right) \bar{\phi}-v \bar{\theta}=-\bar{g}(k, n), \\
\mu\left\{k^{2} \bar{\psi}+\frac{d^{2} \bar{\psi}}{d z^{2}}-2 i k \frac{d \bar{\psi}}{d z}+\alpha\left(\frac{d^{2} \bar{\psi}}{d z^{2}}-k^{2} \bar{\psi}-2 \bar{\omega}_{2}\right)=0,\right. \\
(\gamma+\varepsilon) \frac{d \bar{\omega}_{2}}{d z}+i \beta_{0} k \bar{\phi}=0, \\
\alpha_{0} \frac{d \bar{\Phi}}{d z}-\frac{1}{3} i \beta_{0} k \bar{\omega}_{2}=0, \\
\frac{d \bar{\theta}}{d z}+h \bar{\theta}=0,
\end{gathered}
$$

where $\bar{g}(k, n)$ is the double Fourier transform of $g(x, t)$.

Substitution of $(4.11)-(4.15)$ into $(4.18)-(4.22)$ yields

$$
\begin{aligned}
& q_{1} A+q_{2} B+q_{3} C+q_{4} D=-\bar{g}(k, n), \\
& p_{1} A+p_{2} B+p_{3} C+p_{4} D=0, \\
& r_{3} C+r_{4} D+r_{5} E=0, \\
& t_{3} C+t_{4} D+t_{5} E=0, \\
& s_{1} A+s_{2} B=0
\end{aligned}
$$

where

$$
\begin{array}{ll}
q_{j} & =\left[\begin{array}{ll}
\left.\lambda_{j}{ }^{2}(\lambda+2 \mu)-k^{2} \lambda-\alpha_{j} \nu\right), & j=1,2, \\
-2 i \mu k \lambda_{j}, & j=3,4,
\end{array}\right] \\
p_{j} & =\left[\begin{array}{ll}
-2 i \mu k \lambda_{j}, & j=1,2, \\
\mu\left(k^{2}+\lambda_{j}^{2}\right)+\alpha\left(\lambda_{j}^{2}-k^{2}-2 \alpha_{j}\right), & j=3,4,
\end{array}\right]
\end{array}
$$




$$
\begin{array}{ll}
r_{j}=\left[\begin{array}{ll}
(\gamma+\varepsilon) \alpha_{j} \lambda_{j}, & j=3,4, \\
-i \beta_{0} k, & j=5,
\end{array}\right] \\
s_{j}=\left(\lambda_{j}-h\right) \alpha_{j}, & j=1,2, \\
t_{j}=\left[\begin{array}{ll}
i \beta_{0} k \alpha_{j}, & j=3,4, \\
3 \alpha_{0} \lambda_{j}, & j=5 .
\end{array}\right]
\end{array}
$$

Solving equations (4.23)-(4.27) for $A, B, C, D$ and $E$ we obtain

$$
\begin{gathered}
A=\frac{\Delta_{1}}{\Delta} \bar{g}(k, n), B=\frac{\Delta_{2}}{\Delta} \bar{g}(k, n), C=\frac{\Delta_{3}}{\Delta} \bar{g}(k, n) . \\
D=\frac{\Delta_{4}}{\Delta} \bar{g}(k, n), E=\frac{\Delta_{5}}{\Delta} \bar{g}(k, n),
\end{gathered}
$$

where

and

$$
\begin{aligned}
& \Delta=m_{1}\left(p_{3} m_{5}-q_{3} m_{4}\right)-m_{2}\left(p_{4} m_{5}-q_{4} m_{4}\right), \\
& \Delta_{1}=s_{2}\left(p_{3} m_{1}-p_{4} m_{2}\right), \Delta_{2}=s_{1}\left(p_{4} m_{2}-p_{3} m_{1}\right), \\
& \Delta_{3}=m_{1} m_{4}, \Delta_{4}=-m_{2} m_{4}, \Delta_{5}=m_{3} m_{4},
\end{aligned}
$$

$$
\begin{aligned}
& m_{1}=\left(r_{4} t_{5}-r_{5} t_{4}\right), m_{2}=\left(r_{3} t_{5}-r_{5} t_{3}\right), m_{3}=\left(r_{3} t_{4}-r_{4} t_{3}\right), \\
& m_{4}=\left(s_{1} p_{2}-s_{2} p_{1}\right), m_{5}=\left(s_{1} q_{2}-s_{2} q_{1}\right) .
\end{aligned}
$$

Using the Fourier inverse transformation (4.2) in (4.11) - (4.15) we obtain

$$
\begin{aligned}
& \phi=\frac{1}{2 \pi}-{ }_{-\infty}^{\delta^{\infty}}-\delta^{\infty}\left(A e^{-\lambda} 1^{z}+B e^{-\lambda} 2^{z}\right) e^{-i(k x+n t)} d k d n \text {, } \\
& \theta=\frac{1}{2 \pi}-_{-\infty}^{\rho^{\infty}}{ }_{-\infty}^{\delta^{\infty}}\left(\alpha_{1} A e^{-\lambda 1^{z}}+\alpha_{2} B e^{-\lambda} 2^{z}\right) e^{-i(k x+n t)} d k d n, \\
& \psi=\frac{1}{2 \pi}-{ }_{-\infty}^{\infty} \int_{-\infty}^{\infty}\left(C e^{-\lambda 3^{z}}+D e^{-\lambda} 4^{z}\right) e^{-i(k x+n t)} d k d n \text {, } \\
& \omega_{2}=\frac{1}{2 \pi}-{ }_{-\infty}^{S^{\infty}}{ }_{-\infty}^{S^{\infty}}\left(\alpha_{3} C e^{-\lambda 3^{z}}+\alpha_{4} D e^{-\lambda 4^{z}}\right) e^{-i(k x+n t)} d k d n \text {, } \\
& \Phi=\frac{1}{2 \pi}{ }_{-\infty}^{\rho^{\infty}}{ }_{-\infty}^{\infty} E e^{-\lambda 5^{z}} e^{-i(k x+n t)} d k d n \text {, }
\end{aligned}
$$

Thus, using (4.40)-(4.44) we can obtain the displacement components, force stress, couple stress tensor, vector first moments, and the temperature field in the integral form

$$
\begin{aligned}
& u_{1}=-\frac{1}{2 \pi}-\int_{-\infty}^{\infty} \int^{\infty} u_{1}^{*}(z, k, n) \bar{g}(k, n) e^{-i(k x+n t)} d k d n \text {, } \\
& u_{3}=-\frac{1}{2 \pi}-{ }_{-\infty}^{\infty}{ }_{-\infty}^{\infty} \delta^{\star} u_{3}^{\star}(z, k, n) \bar{g}(k, n) e^{-i(k x+n t)} d k d n \text {, } \\
& \omega_{2}=\frac{1}{2 \pi}-_{-\infty}^{\infty^{\infty}}{ }_{-\infty}^{\infty} \omega_{2}^{*}(z, k, n) \bar{g}(k, n) e^{-i(k x+n t)} d k d n \text {, }
\end{aligned}
$$




$$
\begin{aligned}
& \sigma_{33}=\frac{1}{2 \pi} \int_{-\infty}^{\int^{\infty}}-f^{f^{\infty}} \sigma_{33}^{\star}(z, k, n) \bar{g}(k, n) e^{-i(k x+n t)} d k d n, \\
& \sigma_{31}=\frac{1}{2 \pi}-_{-\infty}^{\infty}-\infty \rho^{\infty} \sigma_{31}^{*}(z, k, n) \bar{g}(k, n) e^{-i(k x+n t)} d k d n \text {, } \\
& \mu_{32}=-\frac{1}{2 \pi}-\infty \rho^{\infty}{ }_{-\infty}^{\infty}{ }^{\star} \mu_{32}(z, k, n) \bar{g}(k, n) e^{-i(k x+n t)} d k d n \text {, } \\
& B_{3} \quad-\frac{1}{2 \pi}-\int_{-\infty}^{\infty}-\oint^{\infty} \beta_{3}^{*}(z, k, n) \bar{g}(k, n) e^{-i(k x+n t)} d k d n \text {, } \\
& \theta=\frac{1}{2 \pi}-\infty \int_{-\infty}^{\infty} \rho^{\star} \stackrel{\star}{\theta}(z, k, n) \bar{g}(k, n) e^{-i(k x+n t)} d k d n \text {, } \\
& \text { where } \\
& u_{1}^{*}(z, k, n)=\frac{1}{\Delta}\left[i k\left(\Delta_{1} e^{-\lambda 1^{z}}+\Delta_{2} e^{-\lambda} 2^{z}\right)+\lambda_{3} \Delta_{3} e^{-\lambda_{3} z}+\lambda_{4} \Delta_{4} e^{-\lambda} 4^{z}\right] \text {, } \\
& u_{3}^{*}(z, k, n)=\frac{1}{\Delta}\left[\lambda_{1} \Delta_{1} e^{-\lambda} 1^{z}+\lambda_{2} \Delta_{2} e^{-\lambda 2}-i k\left(\Delta_{3} e^{-\lambda_{3} z}+\Delta_{4} e^{-\lambda} 4^{z}\right)\right] \text {, } \\
& \omega_{2}^{\star}(z, k, n)=\frac{1}{\Delta}\left[\alpha_{3} \Delta_{3} e^{-\lambda_{3} z}+\alpha_{4} \Delta_{4} e^{-\lambda 4^{z}}\right] \text {, } \\
& \sigma_{33}^{\star}(z, k, n)=\frac{1}{\Delta}\left[\left\{(\lambda+2 \mu) \lambda_{1}^{2}-k^{2} \lambda-v \alpha_{1}\right\} \Delta_{1} e^{-\lambda{ }^{2} z}\right. \\
& +\left\{(\lambda+2 \mu) \lambda_{2}^{2}-k^{2} \lambda-v \alpha_{2}\right\} \Delta_{2} e^{-\lambda 2^{z}} \\
& \left.-2 i \mu k\left(\lambda_{3} \Delta_{3} e^{-\lambda 3^{z}}+\lambda_{4} \Delta_{4} e^{-\lambda} 4^{z}\right)\right] \\
& \sigma_{31}^{\star}(z, k, n)=\frac{1}{\Delta}\left[2 i \mu k\left(\lambda_{1} \Delta_{1} e^{-\lambda_{1} z}+\lambda_{2} \Delta_{2} e^{-\lambda_{2} z}\right)\right. \\
& +\left\{\mu\left(k^{2}+\lambda_{3}^{2}\right)+\alpha\left(\lambda_{3}^{2}-k^{2}-2 \alpha_{3}\right)\right\} \Delta_{3} e^{-\lambda_{3} z} \\
& \left.+\left\{\mu\left(k^{2}+\lambda_{4}^{2}\right)+\alpha\left(\lambda_{4}^{2}-k^{2}-2 \alpha_{4}\right)\right\} \Delta_{4} e^{-\lambda 4^{z}}\right], \\
& \mu_{32}^{*}(z, k, n)=\frac{1}{\Delta}\left[(\gamma+\varepsilon)\left(\lambda_{3} \alpha_{3} \Delta_{3} e^{-\lambda 3^{z}}+\alpha_{4} \lambda_{4} \Delta_{4} e^{-\lambda 4^{z}}\right)-i_{0} k \Delta_{5} e^{-\lambda 5^{z}}\right] \text {, } \\
& \beta_{3}^{\star}(z, k, n)=\frac{1}{\Delta}\left[i \beta_{0} k\left(\alpha_{3} \Delta_{3} e^{-\lambda 3^{z}}+\alpha_{4} \Delta_{4} e^{-\lambda 4^{z}}\right)+3 \alpha_{0} \lambda_{5} \Delta_{5} e^{-\lambda 5^{z}}\right] \text {, } \\
& \theta^{\star}(z, k, n)=\frac{1}{\Delta}\left[\alpha_{1} \Delta_{1} e^{-\lambda 1^{z}} \cdot \alpha_{2} \Delta_{2} e^{-\lambda 2^{z}}\right] \text {. } \\
& \text { 5. PARTICULAR CASES: }
\end{aligned}
$$

(i) We consider a time periodic concentrated force acting at the origin in the direction of $x$-axis so that the loading function assumes the form

$$
g(x, t)=F \delta(x) e^{-i \omega t},
$$

where $F$ is the magnitude of the force, $\delta(x)$ is the Dirac function of distribution and $\omega$ is the frequency.

The double Fourier transform of $g(x, t)$ is 


$$
\begin{aligned}
\bar{g}(k, n) & =\frac{1}{2 \pi}-_{-\infty}^{\infty} \int_{-\infty}^{\infty} F \delta(x) e^{-i \omega t} e^{i(k x+n t)} d x d t, \\
& =\frac{F}{\sqrt{(2 \pi)}}-\infty \int^{\infty} e^{i(n-\omega) t} d t, \\
& =\sqrt{(2 \pi)} F \delta(n-\omega) .
\end{aligned}
$$

Thus from (4.45)-(4.60) with (5.2), we obtain

$$
\begin{aligned}
& u_{1}=-\frac{F}{\sqrt{(2 \pi)}} e^{-i \omega t} \int_{-\infty}^{\infty}\left[u_{1}^{\star}(z, k, n)\right]_{n=\omega} e^{-i k x} d k \text {, } \\
& u_{3}=-\frac{F}{\sqrt{(2 \pi)}} e^{-i \omega t}{ }_{-\infty}^{\rho^{\infty}}\left[u_{3}^{*}(z, k, n)\right]_{n=\omega} e^{-i k x} d k \text {, } \\
& \omega_{2}=\frac{F}{\sqrt{(2 \pi)}} e^{-i \omega t}{ }_{-\infty}^{f^{\infty}}\left[\omega_{2}^{*}(z, k, n)\right]_{n=\omega} e^{-i k x} d k, \\
& c_{33}=\frac{F}{\sqrt{(2 \pi)}} e^{-i \omega t} \int_{-\infty}^{S^{\infty}}\left[\sigma_{33}^{*}(z, k, n)\right]_{n=\omega} e^{-i k x} d k \text {, } \\
& \sigma_{31}=\frac{F}{\sqrt{(2 \pi)}} \mathrm{e}^{-i \omega t}{ }_{-\infty}^{\delta^{\infty}}\left[\sigma_{31}^{*}(z, k, n)\right]_{n=\omega} \mathrm{e}^{-i k x} d k, \\
& \mu_{32}=\frac{F}{\sqrt{(2 \pi)}} e^{-i \omega t}{ }_{-\infty}^{\infty}\left[\mu_{32}^{*}(z, k, n)\right]_{n=\omega} e^{-i k x} d k \text {, } \\
& B_{3}=e-\frac{1}{3} \frac{F}{\sqrt{(2 \pi)}} e^{-i \omega t}{ }_{-\infty}^{\infty}\left[\beta_{3}^{*}(z, k, n)\right]_{n=\omega} e^{-i k x} d k, \\
& \theta=\frac{F}{\sqrt{(2 \pi)}} e^{-i \omega t} \int_{-\infty}^{\infty}\left[\theta^{*}(z, k, n)\right]_{n=\omega} e^{-i k x} d k \text {. }
\end{aligned}
$$

If we neglect the stretch effect, we recover the corresponding expressions for the displacements, stresses, and the temperature field in the form

$$
\begin{aligned}
& u_{1}=-\frac{F e^{-i \omega t}}{\sqrt{(2 \pi)}}{ }_{-\infty} f^{\infty}\left[M_{1}(z, k, n)\right]_{n=\omega} e^{-i k x} d k \text {, } \\
& u_{3}=-\frac{F e^{-i \omega t}}{\sqrt{(2 \pi)}}{ }_{-\infty} f^{\infty}\left[M_{2}(z, k, n)\right]_{n=\omega} e^{-i k x} d k \text {, } \\
& \omega_{2}=\frac{F e^{-i \omega t}}{\sqrt{(2 \pi)}}-f^{f^{\infty}}\left[M_{3}(z, k, n)\right]_{n=\omega} e^{-i k x} d k \text {, } \\
& \sigma_{33}=\frac{F e^{-i \omega t}}{\sqrt{(2 \pi)}}-\int^{j^{\infty}}\left[M_{3}(z, k, n)\right]_{n=\omega} e^{-i k x} d k, \\
& \sigma_{31}=\frac{F e^{-i \omega t}}{\sqrt{(2 \pi)}}-\int^{\delta^{\infty}}\left[M_{3}(z, k, n)\right]_{n=\omega} e^{-i k x} d k, \\
& \sigma_{32}=-\frac{F e^{-i \omega t}}{\sqrt{(2 \pi)}}-\infty^{\infty}\left[M_{1}(z, k, n)\right]_{n=\omega} e^{-i k x} d k, \\
& \theta=\frac{F e^{-i \omega t}}{\sqrt{(2 \pi)}}-_{-\infty}^{f^{\infty}}\left[M_{3}(z, k, n)\right]_{n=\omega} e^{-i k x} d k,
\end{aligned}
$$


where

$$
\begin{aligned}
& M_{1}(z, k, n)=\frac{1}{\Delta^{\star}}\left[i k\left(\Delta_{1}^{*} e^{-\lambda 1^{z}}+\Delta_{2}^{\star} e^{-\lambda} 2^{z}\right)+\lambda_{3} \Delta_{3}^{*} e^{-\lambda 3^{z}}+\lambda_{4} \Delta_{4}^{\star} e^{-\lambda 4^{z}}\right] \text {, } \\
& M_{2}(z, k, n)=\frac{1}{\Delta^{*}}\left[\lambda_{1} \Delta_{1}^{*} e^{-\lambda_{1} z}+\lambda_{2} \Delta_{2}^{*} e^{-\lambda_{2} z}-i k\left(\Delta_{3}^{*} e^{-\lambda_{3} z}+\Delta_{4}^{\star} e^{-\lambda_{4} z}\right)\right] \text {, } \\
& M_{3}(z, k, n)=\frac{1}{\Delta}\left[\alpha_{3} \Delta_{3}^{\star} e^{-\lambda_{3} z}+\alpha_{4} \Delta_{4}^{\star} e^{-\lambda 4^{z}}\right] \text {, } \\
& M_{4}(z, k, n)=\frac{1}{\Delta^{\star}}\left[(\lambda+2 \mu) \lambda_{1}^{2}-k^{2} \lambda-v \alpha_{1}\right\} \Delta_{1}^{\star} e^{-\lambda 2^{z}} \\
& +\left\{(\lambda+2 \mu) \lambda_{2}^{2}-k^{2} \lambda-v \alpha_{2}\right\} \Delta_{2}^{*} e^{-\lambda_{2} z} \\
& \left.-2 i \mu k\left(\lambda_{3} \Delta_{3}^{*} e^{-\lambda 3^{z}}+\lambda_{4} \Delta_{4}^{*} e^{-\lambda 4^{z}}\right)\right] \text {, } \\
& M_{5}(z, k, n)=\frac{1}{\Delta^{\star}}\left[2 i \mu k\left(\lambda_{1} \Delta_{1}^{*} e^{-\lambda 1^{z}}+\lambda_{2} \Delta_{2}^{*} e^{-\lambda_{2} z}\right)\right. \\
& +\left\{\mu\left(k^{2}+\lambda_{3}^{2}\right)+\alpha\left(\lambda_{3}^{2}-k^{2}-2 \alpha_{3}\right)\right\} \Delta_{3}^{*} e^{-\lambda} 3^{z} \\
& \left.+\left\{\mu\left(k^{2}+\lambda_{4}^{2}\right)+\alpha\left(\lambda_{4}^{2}-k^{2}-2 \alpha_{4}\right)\right\} \Delta_{4}^{*} e^{-\lambda} 4^{z}\right], \\
& M_{6}(z, k, n)=\frac{1}{\Delta^{\star}}\left[(\gamma+\varepsilon)\left(\lambda_{3} \alpha_{3} \Delta_{3}^{*} e^{-\lambda_{3} z}+\lambda_{4} \alpha_{4} \Delta_{4}^{*} e^{-\lambda_{4} z}\right)\right], \\
& M_{7}(z, k, n)=\frac{1}{\Delta}\left[\alpha_{1} \Delta_{1}^{*} e^{-\lambda 1^{z}}+\alpha_{2} \Delta_{2}^{*} e^{-\lambda 2^{z}}\right],
\end{aligned}
$$

and

$$
\begin{aligned}
& \Delta^{*}=\left(p_{3} r_{4}-p_{4} r_{3}\right)\left(s_{2} q_{1}-s_{1} q_{2}\right)-\left(q_{3} r_{4}-q_{4} r_{3}\right)\left(s_{2} p_{1}-s_{1} p_{2}\right), \\
& \Delta_{1}^{*}=-s_{2}\left(p_{3} r_{4}-p_{4} r_{3}\right), \\
& \Delta_{2}^{*}=s_{1}\left(p_{3} r_{4}-p_{4} r_{3}\right), \\
& \Delta_{3}^{*}=-r_{4}\left(s_{1} p_{2}-s_{2} p_{1}\right), \\
& \Delta_{4}^{*}=r_{3}\left(s_{1} p_{2}-s_{2} p_{1}\right) .
\end{aligned}
$$

These results agree with those obtained by Acharya and Sengupta [8].

(ii) In this case we consider a torque with its axis parallel to the z-axis so that $g(x, t)$ can be written as

$$
g(x, t)=G[\delta(x-a)-\delta(x+a)] e^{-i \omega t}
$$

where $G$ is the magnitude of the force.

The double Fourier transformation of $(5.30)$ gives

$$
\begin{aligned}
\bar{g}(k, n) & =\frac{G}{2 \pi}-{ }_{-\infty} \delta^{\infty}-\infty \delta^{\infty}[\delta(x-a)-\delta(x+a)] e^{i(k x+n t)} e^{-i \omega t} d x d t, \\
& =2 i \sqrt{(2 \pi)} G \sin (k a) \delta(n-\omega) .
\end{aligned}
$$


Then from (4.45) - (4.60) with (5.31), we obtain

$$
\begin{aligned}
& u_{1}=-i G \sqrt{(2 / \pi)} e^{-i \omega t} \int_{-\infty}^{\infty}\left[u_{1}^{*}(z, k, n)\right]_{n=\omega} \sin (k a) e^{-i k x} d k \text {, } \\
& u_{3}=-i G \sqrt{(2 / \pi)} e^{-i \omega t}-\infty f^{\infty}\left[u_{3}^{*}(z, k, n)\right]_{n=\omega} \sin (k a) e^{-i k x} d k, \\
& \omega_{2}=i G \sqrt{(2 / \pi)} e^{-i \omega t} \int_{-\infty}^{\infty}\left[\omega_{2}^{*}(z, k, n)\right]_{n=\omega} \sin (k a) e^{-i k x} d k \text {, } \\
& \sigma_{33}=i G \sqrt{(2 / \pi)} e^{-i \omega t} \int_{-\infty}^{\infty}\left[\sigma_{33}^{*}(z, k, n)\right]_{n=\omega} \sin (k a) e^{-i k x} d k, \\
& \sigma_{31}=i G \sqrt{(2 / \pi)} e^{-i \omega t}{ }_{-\infty}^{\infty}\left[\sigma_{31}^{*}(z, k, n)\right]_{n=\omega} \sin (k a) e^{-i k x} d k, \\
& \mu_{32}=-i G \sqrt{(2 / \pi)} e^{-i \omega t} \rho_{-\infty}^{\infty}\left[\mu_{32}^{\star}(z, k, n)\right]_{n=\omega} \sin (k a) e^{-i k x} d k \text {, }
\end{aligned}
$$

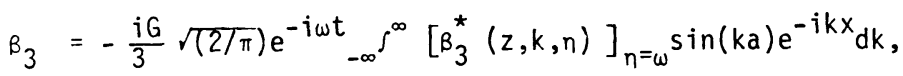

$$
\begin{aligned}
& \theta=i G \sqrt{(2 / \pi)} e^{-i \omega t} \int_{-\infty}^{5^{\infty}}\left[\theta^{*}(z, k, n)\right]_{n=\omega} \sin (k a) e^{-i k x} d k \text {. }
\end{aligned}
$$

In the absence of the stretch effect, we obtain the corresponding expressions for the displacements, stresses and the temperature field in the form

$$
\begin{aligned}
& u_{1}=-i G \sqrt{(2 / \pi)} e^{-i \omega t} \int_{-\infty}^{\delta^{\infty}}\left[M_{1}(z, k, n)\right]_{n=\omega} \sin (k a) e^{-i k x} d k, \\
& u_{3}=-i G \sqrt{(2 / \pi)} e^{-i \omega t}-\infty \int^{\infty}\left[M_{2}(z, k, n)\right]_{n=\omega} \sin (k a) e^{-i k x} d k \text {, } \\
& \omega_{2}=i G \sqrt{(2 / \pi)} e^{-i \omega t} \int_{-\infty}^{\infty}\left[M_{3}(z, k, n)\right]_{n=\omega} \sin (k a) e^{-i k x} d k \text {, } \\
& \sigma_{33}=i G \sqrt{(2 / \pi)} e^{-i \omega t} \int_{-\infty}^{S^{\infty}}\left[M_{4}(z, k, n)\right]_{n=\omega} \sin (k a) e^{-i k x} d k \text {, } \\
& \sigma_{31}=i G \sqrt{(2 / \pi)} e^{-i \omega t} \int_{-\infty}^{\rho^{\infty}}\left[M_{5}(z, k, n)\right]_{n=\omega} \sin (k a) e^{-i k x} d k \text {, } \\
& \mu_{32}=-i G \sqrt{(2 / \pi)} e^{-i \omega t}{ }_{-\infty}^{\infty}\left[M_{6}(z, k, n)\right]_{n=\omega} \sin (k a) e^{-i k x} d k \text {, } \\
& \theta=i G \sqrt{(2 / \pi)} e^{-i \omega t}{ }_{-\infty}^{\infty}\left[M_{7}(z, k, n)\right]_{n=\omega} \sin (k a) e^{-i k x} d k \text {. }
\end{aligned}
$$

These results also agree with the corresponding results without stretch.

6. CONCLUSION.

The displacement field, force stress, couple stress, temperature field and vector first moment have been obtained. It is noted that the displacement field, force stress, couple stress and temperature field involve the parameters $\alpha_{0}, \beta_{0}$ and $n_{0}$ of the micropolar elastic media with stretch. In addition to the displacements, force stress, couple stress, and temperature field, vector first moment $\beta_{j}$ has been determined which vanishes in the case of thermo-micropolar elasticity. Some numerical calculation for specific models of physical interest will be carried out and will be communicated in a subsequent paper. 
Acknowledgement: The second author expresses his grateful thanks to Guru Nanak Dev University, Amritsar for providing financial assistance during the preparation of the paper.

\section{REFERENCES}

1. Eringen, A. C., and Suhubi, E. S. Nonlinear Theory of Simple Micro Elastic Solids I, Internal Jour. Eng. Sci. 2 (1964) 189-203; Nonlinear Theory of Micro-elastic Solid II, Internal Jour. Eng. Sci 2 (1964) 389-404.

2. Eringen, A. C. Linear Theory of Micropolar Elasticity, J. Math. Mech 15 (1966) 909-924.

3. Eringen, A. C. Micropolar Fluids with Stretch, Internal Jour Eng. Sci $\geq$ (1969) $115-127$.

4. Eringen, A. C. Ari Kitabevi Math No. 24 (1971)

5. Lamb, H. On the Propagation of Tremors over the Surface of Elastic Solid, Phil. Trans. Roy. Soc, London, A203 (1904) 1-42.

6. Nowacki, W. and Nowacki, W. K. The Plane Lamb's Problem in a Semi-Infinite Micropolar Elastic Body, Arch Mech Stos 21 (1969) 241-251.

7. Chadha, T. K. Plane Lamb's Problem in Micropolar Elasticity, Gerlands Beitr., Geophysik, Leipzig 88 (1979) 407-414.

8. Acharya, D., and Sengupta, P. R. Lamb's Plane Problem in Micropolar-Elastic Medium under Influence of Temperature, Bull Polish Acad. Sci Series Sci Tech 27 (1979) 419-427.

9. Nowacki, W. Theory of Micropolar Elasticity, International Centre for Mechanical Sciences, Courses and Lectures No. 25, Springer Verlag, Berlin (1970) 


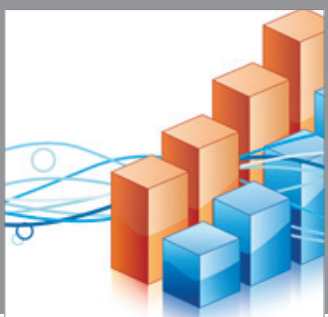

Advances in

Operations Research

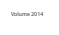

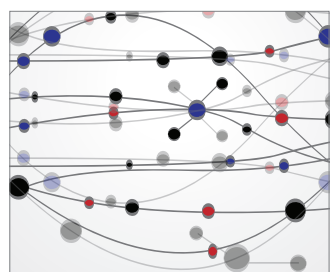

\section{The Scientific} World Journal
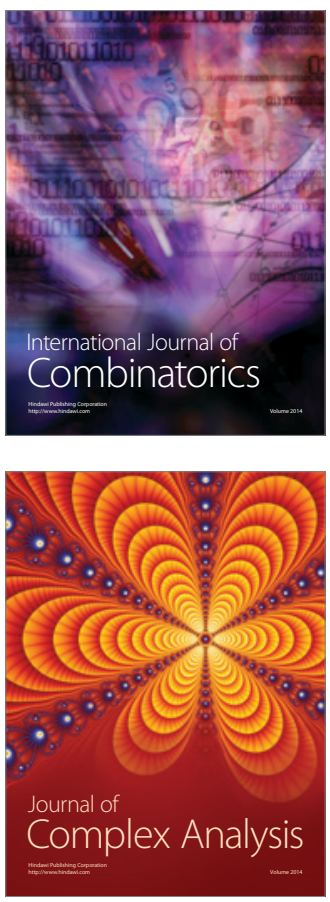

International Journal of

Mathematics and

Mathematical

Sciences
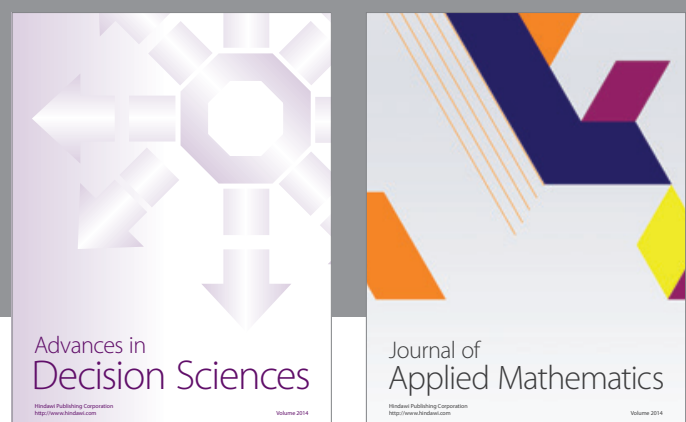

Journal of

Applied Mathematics
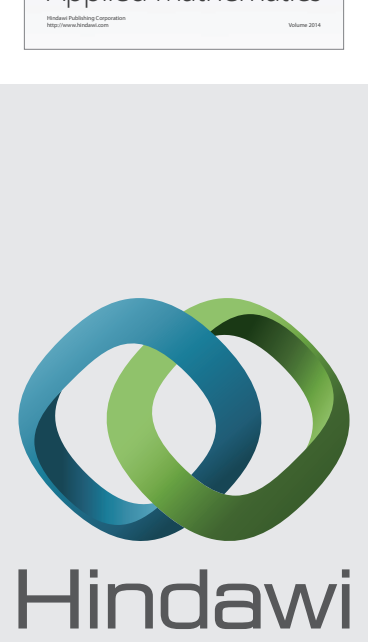

Submit your manuscripts at http://www.hindawi.com
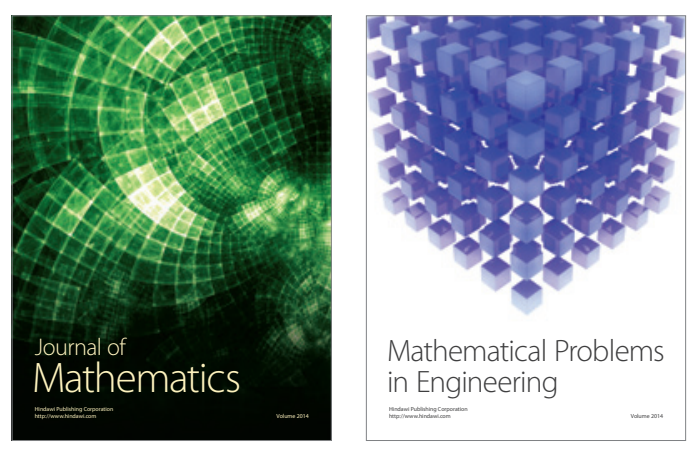

Mathematical Problems in Engineering
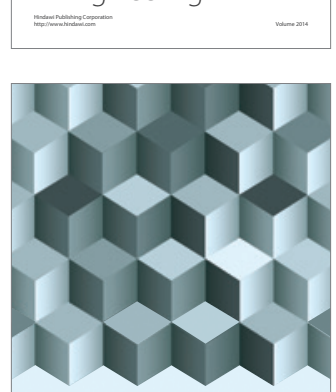

Journal of

Function Spaces
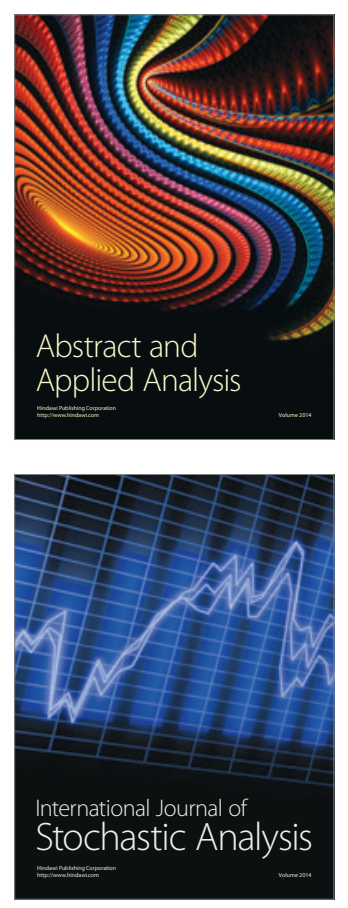

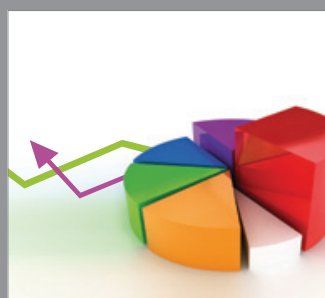

ournal of

Probability and Statistics

Promensencen
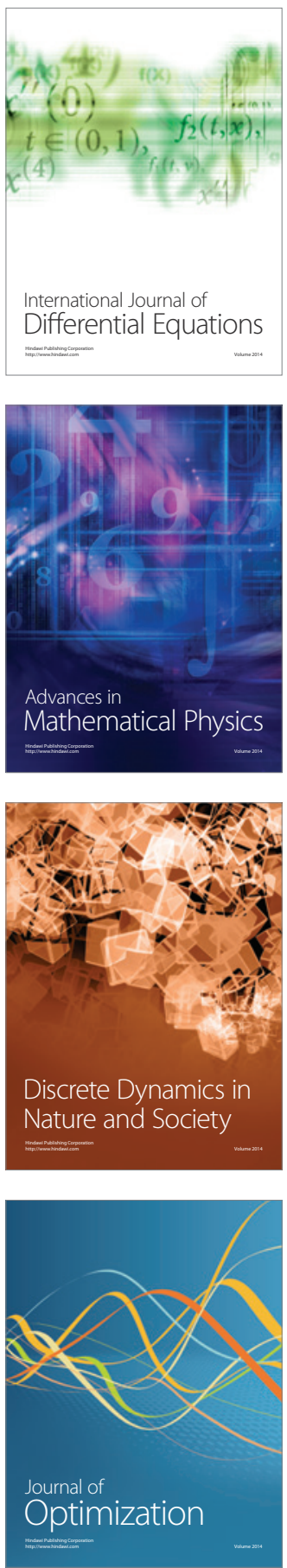